\title{
Safeguarding Wild Plant Genetic Resources of Georgia within the Millennium Seed Bank Partnership
}

\author{
Tsira Mikatadze-Panstulaia ${ }^{1}$, Sandro Kolbaia ${ }^{2}$ and Ana Gogoladze ${ }^{3}$
}

\begin{abstract}
Working group of the Department of Plant Conservation of the National Botanical Garden of Georgia (NBGG) have been participating in the global Millennium Seed Bank Partnership, led by the Royal Botanic Gardens, Kew since 2005. During the 2005-2018 period, within the scope of MSB-1 and MSB-2, seeds and herbarium samples of more than 1750 plant species and interspecific taxa, belonging to 107 families and 483 genera (more than $41 \%$ of Georgia's flora) - 348 endemics of Caucasus and 151 endemics of Georgia, have been secured in the National Seed Bank of Georgia (NSB). Seed Bank data are managed in BRAHMS (Department of Plant Sciences of Oxford University). The collection of wild plant species is accompanied by the comprehensive database of geographical, botanical and habitat information. Later phase involves laboratory treatment and germination/viability testing (at least 500 seeds per species) and the long-term deposition and storage (under $-20^{\circ} \mathrm{C}$ temperature) at the National Seed Bank of Georgia. The duplicates of seed collection and herbarium vouchers are stored at the Millennium Seed Bank of Royal Botanic Gardens, Kew, UK. Germination capacity and viability of collections in NSB is determined before cold storage of seeds, while at the MSB already banked seeds are tested.
\end{abstract}

Keywords: Seed bank; Ex-situ conservation; Plant diversity; Botanical garden; Genetic resources

\section{Introduction}

The republic of Georgia has one of the highest concentration of plant species know from any temperate part of the world. Bordered to the north by Russian Federation, to the east by Azerbaijan, to the south by Armenia and Turkey and to the west by the Black Sea, Georgia's $69,700 \mathrm{~km}^{2}$ are home to an exceptional plant diversity in an area where the European and Asian floras intersect. The Greater Caucasus Mountains traverse northern Georgia, the Lesser Caucasus Mountains cross the southern edge and a rich central valley is bounded by the dry Iori Plateau and Alazani Valley to the east and the humid Kolkheti Plain to the west. This topographic diversity, including mountain peaks that exceed 5,000 meters (Mt. Shkhara, Mt. Mkinvartsveri), coupled with a diverse array of climatic influences, gives rise to a complex mosaic of vegetation zones that support a high diversity of plants.

Georgian flora comprises of the vascular plants grown in wild conditions on the territory of country, comprising 185 families, 1048 genera and 4275 species. High endemism level of Georgian flora reflects its richness - $21 \%$ of the local flora is endemic and consists of about 900 species. Among them about 600 species are endemic to the Caucasus and about 300 are endemic to Georgia (Davliandize et al. 2018)

\footnotetext{
${ }^{1}$ Head of the Department of Plant Conservation of the National Botanical Garden of Georgia, Tbilisi, Georgia. Head of National Seed Bank of Georgia

${ }^{2}$ Research Assistant at the Department of Plant Conservation of the National Botanical Garden of Georgia, Tbilisi, Georgia

${ }^{3}$ Research Assistant at the Department of Plant Conservation of the National Botanical Garden of Georgia, Tbilisi, Georgia
} 
The Caucasus, and Georgia in particular, has been attributed to the world's biodiversity hotspots.

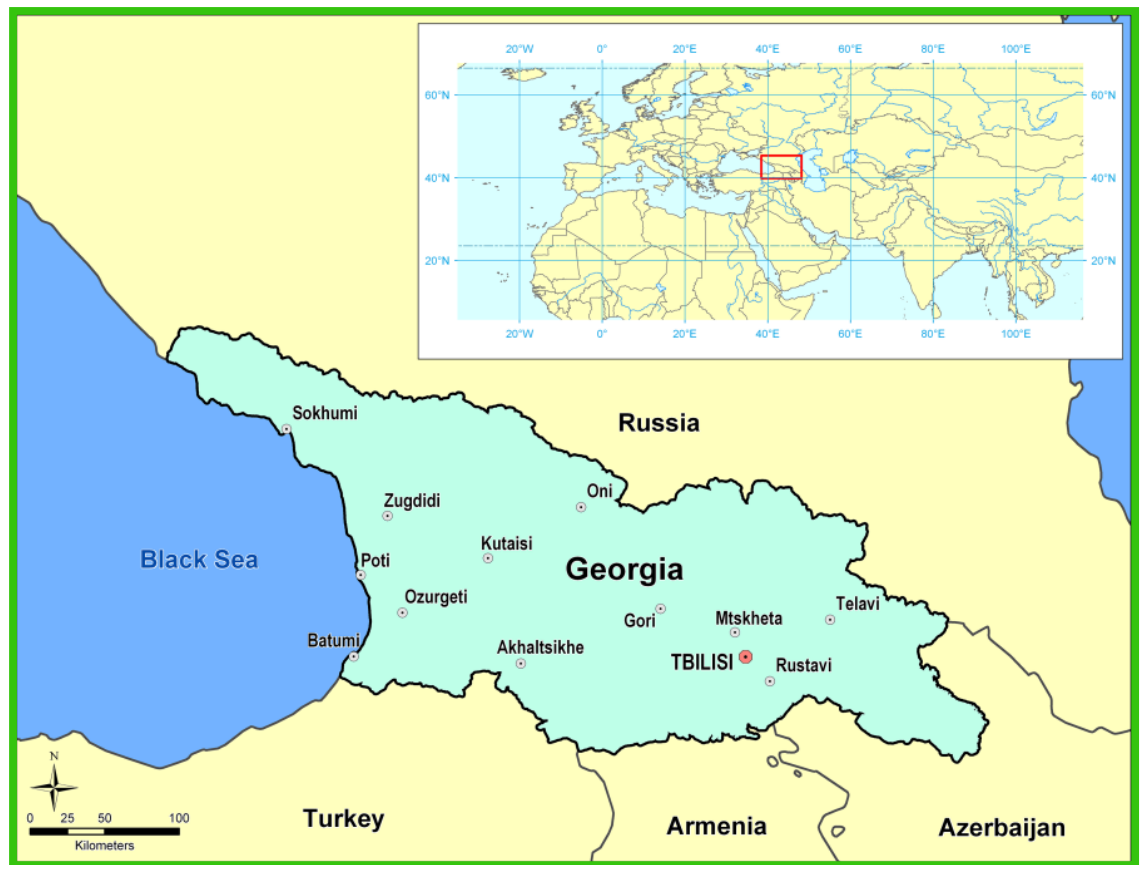

Figure 1: The Republic of Georgia on the world map.

Ex-situ conservation via establishing seed banks and living collections is one of the most efficient and comparatively low-cost method for the protection of plant biodiversity.

The seed bank was founded on behalf of Caucasus Regional Seed Bank (CRSB) in Georgia in 2001 by the staff members of the N. Ketskhoveli Institute of Botany in collaboration with colleagues from the Missouri Botanical Garden (USA) with financial support from the CRDF (U.S. Civilian Research \&Development Foundation). Two joint projects: "Informatics for the Sustainable Use of Plant Genetic Resources in the Republic of Georgia" (2001-2002) and "Sustainable Use \& Conservation of Medicinal Plant Resources in the Republic of Georgia" (2004-2006) were accomplished in collaboration with American colleagues. As a result, seeds of 150 endemic and rare species were deposited in the seed bank, the database created using the information system TROPICOS and the results published in Miller 2006.

The Caucasus Regional Seed Bank has been renamed as The National Seed Bank of Georgia (NSB) under The National Botanical Garden's Plant Conservation Department. The former comprises of 2 ha of collection plot with living plants from the Caucasus.

As of partnership with the Millennium Seed Bank of the Kew Botanical Garden: The global project - The Millennium Seed Bank Partnership (MSB) was launched by the Seed Conservation Department of the Royal Botanic Gardens, Kew in 2000. Target of the first stage of the project (MSB-1), that was completed in March of 2010, was to bank the seeds of $10 \%$ of the vascular flora of the world (all species from the United Kingdom as 
well as nearly 24,200 mainly dryland species) at a new storage facility located in the Wakehurst Place, West Sussex, later called the Welcome Trust Millennium Building. The project was also aimed at the development of partnership with organisations in many countries, bilateral research, training and capacity building for the support of plant conservation at a global scale.

Scientists of the Department of Plant Conservation of the N. Ketskhoveli Institute of Botany joined this global initiative in 2005 with the project "Ex-situ conservation of the wild flora of Georgia in the Caucasus Regional Seed Bank (Georgia) and the Millennium Seed Bank (UK) 2005-2010 (N4714 P4/6, 22 August, 2005). Georgia was the first former Soviet country to join the MSB-1. Priority for the project was creation of the seed bank of endemic, rare, threatened, vulnerable and economically important plants.

MSB-1 was completed in March 2010. During the period of 2005-2010, in collaboration with British colleagues within the scope of the MSB-1, seeds and herbarium samples of 848 plant species have been collected, comprising around $21 \%$ of Georgia's flora (Mikatadze-Pantsulaia et al. 2010).

MSB-2 or MSBP (Millennium Seed Bank Partnership), as is referred the second decade of this global project (2010-2020), aims at conserving seeds of another 15\% of world's seed-bearing plants at the Millennium Seed Bank during the current decade and enabling practical use of these seeds for the well-being of mankind.

Staff members of the Department of Plant Conservation of the National Botanical Garden of Georgia together with colleagues from the Institute of Botany of the Ilia State University continue their work under the project „Saving the Flora of the Caucasus” to achieve targets of the second stage of the MSBP (2010-2020).

\section{Materials and Methods}

Seeds of target species are to be necessarily collected in the wild. For each species a special data sheet is filled out, which, along with geographic and botanical data, contains comprehensive information about the sample, its habitat and the process of collecting. After the primary processing all these data are transferred into the electronic database - BRAHMS (Botanical Research and Herbarium Management System) and is electronically exchanged with the MSB and MSB DW (Data Warehouse).

The National Seed Bank (NSB) is designed for the long-term storage of seeds. All seed bank procedures are in accordance with international standards and guidelines (Baskin 2001; Smith 2003).

Upon arrival to the seed bank seeds are dried in dry room and then processed (manual cleaning, sorting, removal of infested, damaged or empty seeds, cleaned (if appropriate) using the aspirator (CB-1 Column Blower, Agriculex Inc., Canada), weighted and numbered. Specialists carefully check seed maturity and collection quality - the collection is visually evaluated using the binocular stereoscope (Motic SMZ-161, Hong Kong). Depending on the size of a collection, certain number of seeds are cut tested, as well as, in some cases, stained with tetrazolium chloride ( $T Z$ test) for the evaluation of their viability. After the examination and testing of seeds, "adjusted seed number" is determined, considering the percentage of potentially non-viable seeds (empty, halffilled, damaged or infested). Before banking seeds are kept in the dry room at the relative 
humidity $(\mathrm{rH})$ of $15-20 \%$. Above indicated equilibrium relative humidity is safe level to store seeds at the minus temperature. Dried seeds are placed in aluminium foil bags, sealed using the sealing apparatus (Hulme Martin Heat Sealers Ltd, UK) and banked in the freezer at $-20^{\circ} \mathrm{C}$. Germination capacity of seeds is tested on Petri dishes on $1 \%$ plain agar medium inside the incubator (LMS Ltd, UK) under controlled illumination and temperature regime. Results and conditions of germination tests along with the data of seed processing (cleaning, weighing, cut testing) and relative humidity measurements are transferred to the electronic database (BRAHMS), which automatically calculates percentages of germination and viability.

Germination capacity and viability of collections in NSB is determined before cold storage of seeds, while at the MSB already banked seeds are tested for germination every ten years.
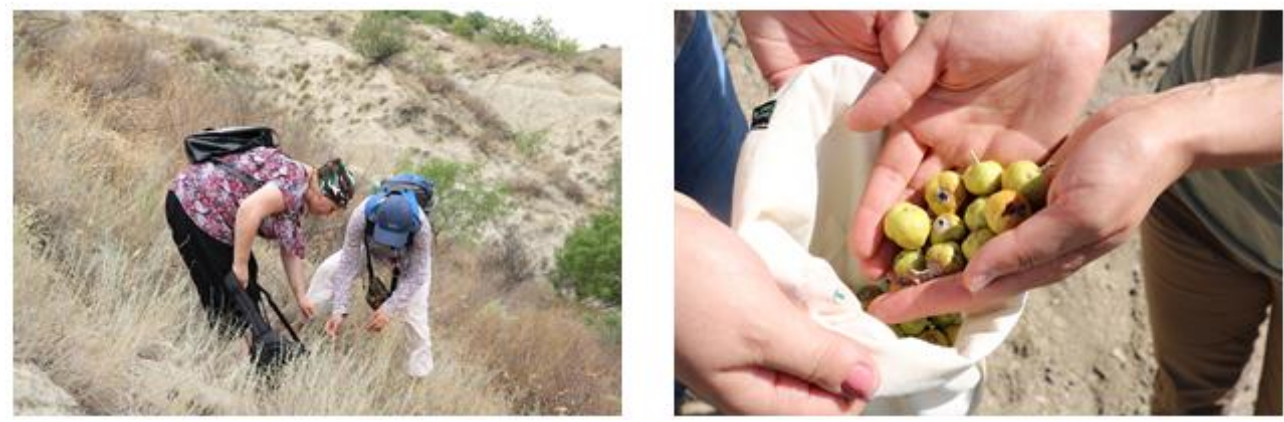

Figures 2, 3: NSB team members assessing the population of target species - Eversmannia subspinosa (DC.) B.Fedtsch. in Vashlovani Nature Reserve, Eastern Georgia (left). Collection process of Caucasian endemic pear species Pyrus takhtadz̧bianii Fed. in Eastern Georgia (right).

\section{Conclusion and Discussion}

During the 2005-2018 period, within the scope of MSB-1 and MSB-2, seeds and herbarium samples of more than 1750 plant species and interspecific taxa, belonging to 107 families and 483 genera (more than $41 \%$ of the Georgian flora) - 348 endemics of Caucasus and 151 endemics of Georgia, have been secured in the National Seed Bank of Georgia (NSB). Among them are 134 species of woody plants, as well as 168 ssp. of crop wild relatives (CWR) from 298 populations.

The information gathered in the seed bank is managed using the BRAHMS database.

Minimum number of 500 seeds from each species are deposited at the NSB and the corresponding labelled herbarium specimen kept at the National Herbarium of Georgia (TBI). The duplicates of the seed collection and herbarium voucher are sent to the Millennium Seed Bank and Herbarium of the Royal Botanic Gardens, Kew (K). Under the partnership, the "excess" seeds of the plants of conservation importance are used for building the documented living collections for later use in in-situ reintroduction works, scientific research and public awareness. 


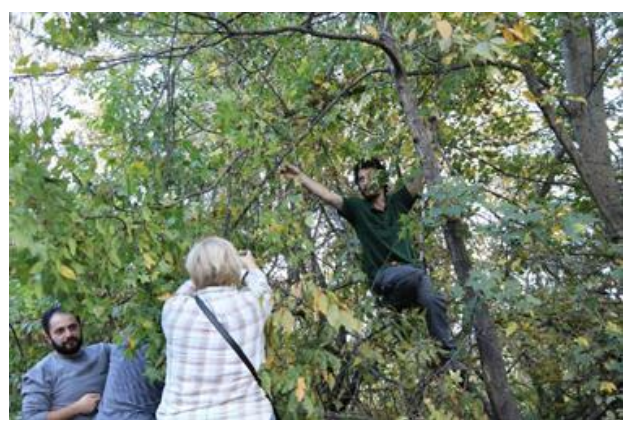

Figure 4: Seed collecting process of Acer laetum C.A.Mey. at Gombori pass in Eastern Georgia

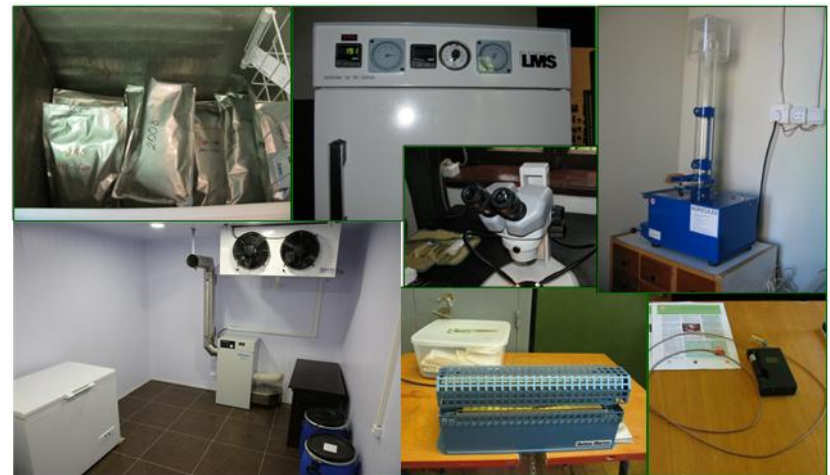

Figure 5: National Seed Bank facility and equipment.

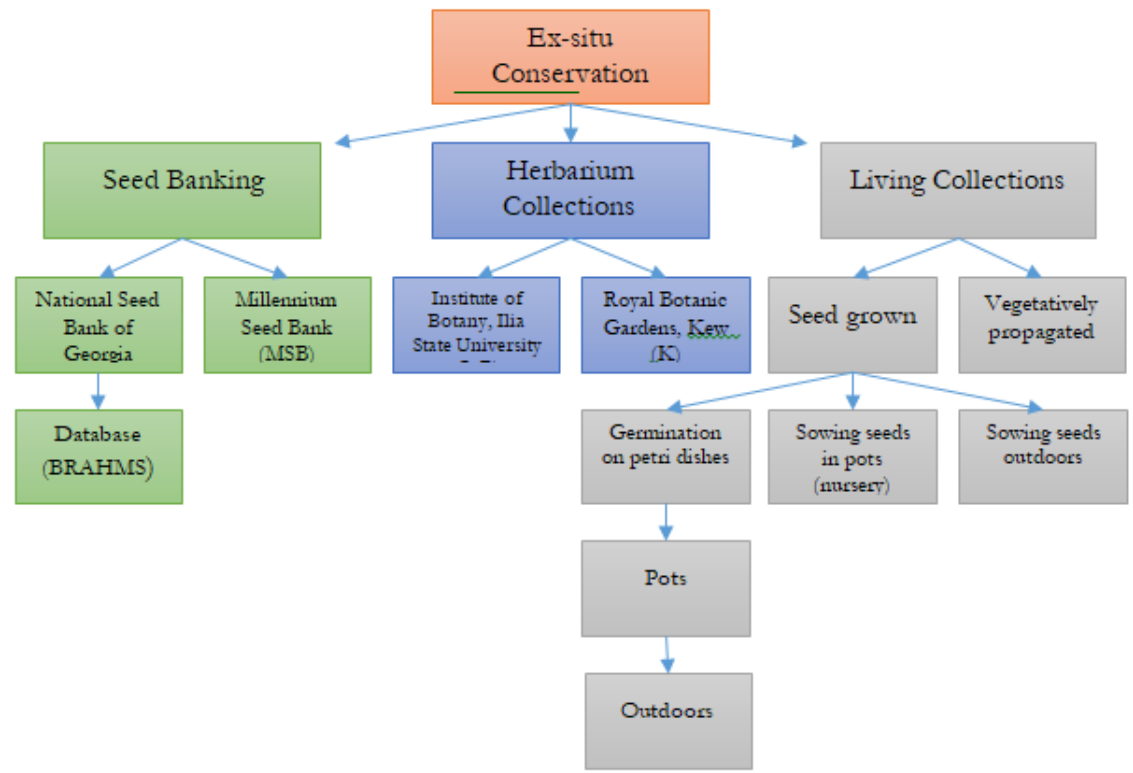

Figure 6: Structure of ex-situ conservation work at the National Seed Bank (NSB) within the Millennium Seed Bank partnership (MSB). 


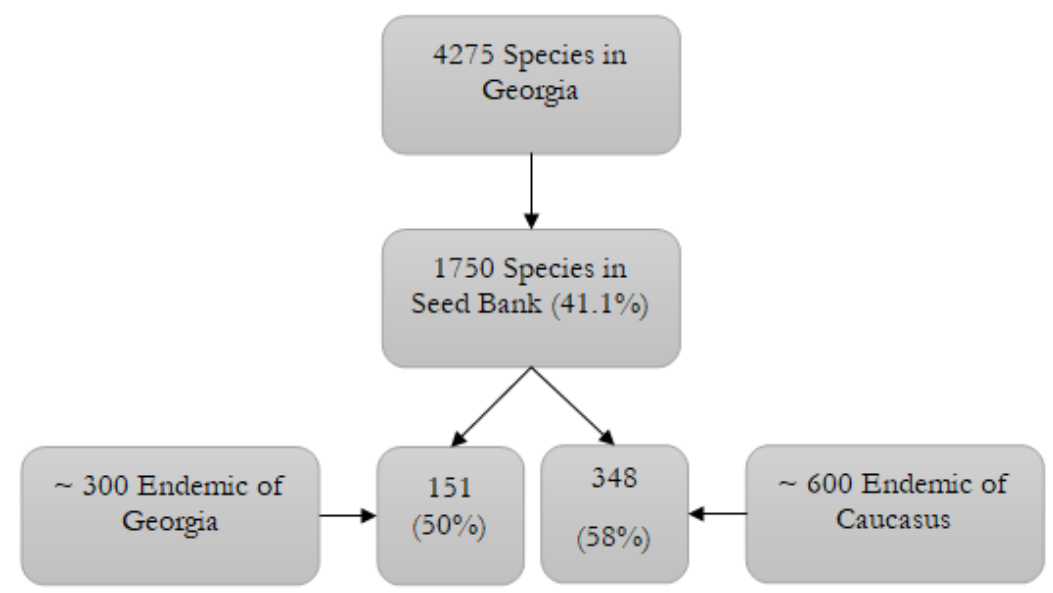

Figure 7: Current state of collections at the National Seed Bank (2005-2018).

\section{Acknowledgements}

We thank: Staff of the Millennium Seed Bank of the Royal Botanic Gardens, Kew (UK), the Rufford Foundation (UK) and personally Mr. David Hunt (BritishGeorgian Society, UK).

\section{References}

Baskin, C. C. \& Baskin, J.M. (2001) Seeds, Ecology, Biogeography and Evolution of Dormancy and Germination. Academic Press, California.

Davlianidze M. and et al. (2018) Nomenclatural Checklist of Flora of Georgia. Ilia State University Institute of Botany, Tbilisi

Miller J.S., McCue K., Consiglio T., Stone J., Eristavi M., Sikharulidze Sh., Mikatadze-Pantsulaia Ts., Khutsishvili M. (2006) Endemic Medicinal Plants of Georgia (Caucasus). 45p.

Mikatadze-Pantsulaia Ts., Barblishvili T., Trivedi C., Kikodze D., Khutsishvili M. (2010) Ex-situ conservation of some endemic and protected plant species in Georgia, Kew Bulletin, 65 (4):643448.

Mikatadze-Pantsulaia Ts., Barblishvili T., Trivedi C., Faruk A., (2017) Caucasus Regional Seed Bank, Nova Science Publishers Inc. New York, USA

Solomon J., Shulkina T., Schatz E. G. (2014) Red List of the Endemic Plants of the Caucasus: Armenia, Azerbaizan, Georgia, Iran, Russia, and Turkey. Monographs in Systematic Botany from Missouri Botanical

Smith, R. D., Dickie, J. B., Linington, S. H., Pritchard, H. W., \& Probert, R. J. (2003). Seed Conservation: Turning Science into Practice. London. The Royal Botanic Gardens, Kew.

Trivedi C. (2012) Seed scientists and horticulturalists working together to conserve threatened Georgian species. Samara, 23 July-December 